\title{
Weak localization and interaction effects in acceptor graphite intercalation compounds
}

Article in Low Temperature Physics · June 2017

DOI: $10.1063 / 1.4985977$

CITATIONS

0

8 authors, including:

\section{Ludmila Matzui}

National Taras Shevchenko University of Kyiv 77 PUBLICATIONS 343 CITATIONS

SEE PROFILE

\section{Igor B Berkutov}

North Carolina State University

50 PUBLICATIONS 183 CITATIONS

SEE PROFILE
READS

10
T. A. Len

National Taras Shevchenko University of Kyiv 17 PUBLICATIONS 83 CITATIONS

SEE PROFILE

Francois Le Normand

University of Strasbourg

275 PUBLICATIONS 2,929 CITATIONS

SEE PROFILE

Some of the authors of this publication are also working on these related projects:

Project Electron transport in new conducting and superconducting systems View project

Project Material science View project 


\section{Weak localization and interaction effects in acceptor graphite intercalation compounds}

O. I. Prokopov, I. V. Ovsiienko, L. Yu. Matzui, T. A. Len, D. D. Naumova, I. B. Berkutov, I. G. Mirzoiev, and F. Le Normand

Citation: Low Temperature Physics 43, 703 (2017); doi: 10.1063/1.4985977

View online: http://dx.doi.org/10.1063/1.4985977

View Table of Contents: http://aip.scitation.org/toc/ltp/43/6

Published by the American Institute of Physics

\section{Articles you may be interested in}

Nonlinear current resonance in a spin-torque diode with planar magnetization

Low Temperature Physics 43, 708 (2017); 10.1063/1.4985978

The study of chromium borate $\mathrm{CrBO}_{3}$ by optical and IR spectroscopy

Low Temperature Physics 43, 728 (2017); 10.1063/1.4985982

Oscillations of a single Abrikosov vortex in hard type-II superconductors

Low Temperature Physics 43, 670 (2017); 10.1063/1.4985973

The effect of depairing on the current-phase relation in SIS junctions in the presence of nonmagnetic impurities of arbitrary concentration

Low Temperature Physics 43, 664 (2017); 10.1063/1.4985972

Embedded soliton dynamics in the asymmetric array of Josephson junctions

Low Temperature Physics 43, 658 (2017); 10.1063/1.4985986

The energy spectrum of zigzag single-walled carbon nanotubes in the Hubbard model, in the static-fluctuation approximation

Low Temperature Physics 43, 719 (2017); 10.1063/1.4985980

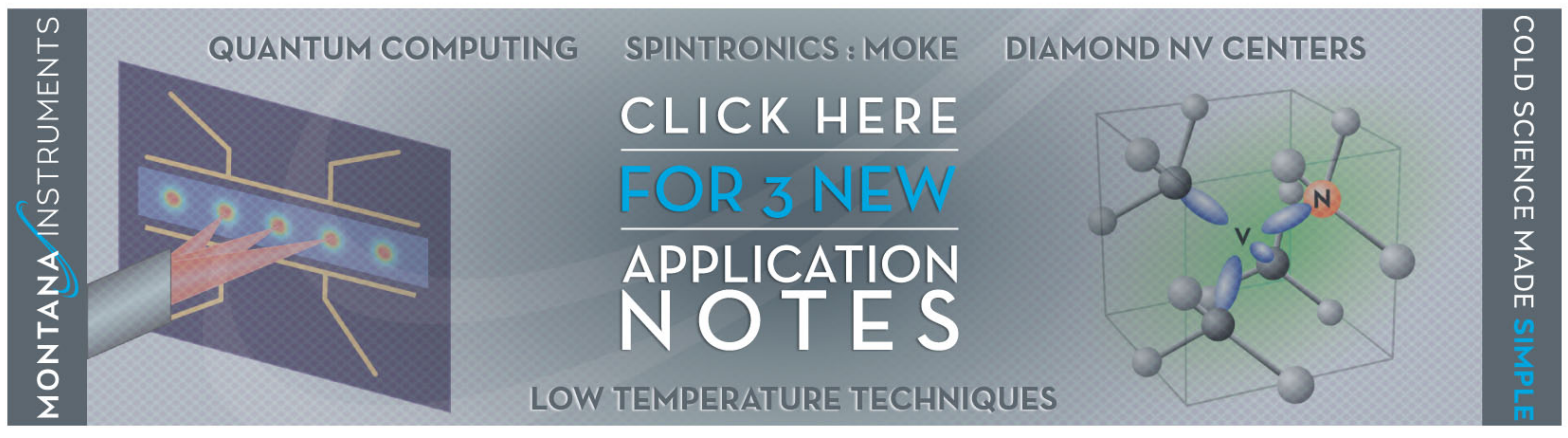




\title{
Weak localization and interaction effects in acceptor graphite intercalation compounds
}

\author{
O. I. Prokopov, I. V. Ovsiienko, L. Yu. Matzui, ${ }^{a)}$ and T. A. Len \\ Department of Physics, Taras Shevchenko National University of Kyiv, 64/13 Volodymyrska Str., Kyiv 01601, \\ Ukraine

\section{D. Naumova}

Department of Chemistry, Taras Shevchenko National University of Kyiv, 64/13 Volodymyrska Str., Kyiv 01601, Ukraine

\section{B. Berkutov ${ }^{\text {b) }}$ and I. G. Mirzoiev}

B. Verkin Institute for Low Temperature Physics and Engineering, National Academy Sciences of Ukraine, 47 Nauky Ave., Kharkiv 61103, Ukraine

\section{F. Le Normand}

Institut de Physique et Chimie des Matériaux, 23 rue du Loess BP 43, Strasbourg 67037, France (Submitted August 12, 2016)

Fiz. Nizk. Temp. 43, 884-888 (June 2017)

\begin{abstract}
The presented work is devoted to investigations of manifestation of quantum effects of weak localization and interaction of charge carriers in electrical conductivity of acceptor graphite intercalation compounds (CICs). As shown by studies intercalation leads to a decrease in the resistivity and to change the resistivity temperature coefficient from negative sign in the source graphite on a positive sign in intercalated graphite. At the low temperature for all GICs specimens the minimum in the temperature dependence of resistivity is observed. In terms of the model of charge carrier's weak localization and interaction for two-dimensional systems temperature dependence of phase relaxation time, localization radius and charge carriers screening constant for all GICs are estimated. Published by AIP Publishing. [http://dx.doi.org/10.1063/1.4985977]
\end{abstract}

\section{Introduction}

The theory of charge carriers weak localization and interactions was developed for disordered metals, semimetals and degenerate semiconductors. ${ }^{1-3}$ As expected in Mott theory the transition metal-insulator occurs abruptly. The theory of weak localization assumed that the transition from metallic to insulator state, in which the electron wave function decreases exponentially, is not abrupt. There is an intermediate region of structures in which the electron wave function decreases slowly. A charge carriers weak localization is due to interference of direct and elastically scattered backwards on the inhomogeneities of structure electronic waves. In the theory of electron-electron interaction inelastic interactions of electrons with each other and with phonons or other degrees of freedom is taken into account. If the condition $k_{F} L \sim 1$ occurs the character of electron-electron interaction is qualitatively changed, because during the interaction time electrons are scattered repeatedly at impurities or defects. Quantum effects of charge carriers weak localization and interactions lead to the appearance of abnormal temperature and magnetic field dependences of transport properties. In first abnormal low-temperature dependence of electrical resistance is observed for fine crystalline graphite in Refs. 4 and 5. It was shown that a sharp proportional to $T^{1 / 2}$ increase of electrical resistance for fine crystalline graphite can be explained in the terms of theory of weak localization and interaction of charge carriers. As another manifestation of quantum effects in this material negative magnetoresistance in fine crystalline graphite was found.
As it was shown in several papers there are quantum effects of weak localization and interaction of charge carriers in the multiwall carbon nanotubes. ${ }^{6-9}$ More pronounced weak localization and interaction effects are for two-dimensional systems. Graphite intercalation compounds (GICs) are natural two-dimensional electronic systems in which carriers move mainly in a direction parallel to the planes of graphite, so the charge carriers system in GICs of low stages can be seen as degenerate dimensional electron gas, two-dimensionality of which is associated with structural features of the electronic structure of GICs.

Abnormal transport properties were revealed for GICs based on graphite fibers and high oriented pyrolitic graphite (HOPG) also. ${ }^{10-13}$ These anomalies authors are associated with the manifestation of the weak localization and interaction effects.

However, despite a number of researches on these effects in GICs, question about the ratio between contributions of weak localization and interaction effects is open. Also conclusion on observation of weak localization and interaction effects in the GIC based on HOPG is ambiguous. So, the aim of this work is to investigate the low-temperature anomalies in resistivity of GICs based on graphites with different structure perfection and to establish the nature of these anomalies.

\section{Experimental results and discussion}

As source for intercalation two types of graphite with different structure perfection were used. These are highly oriented pyrolytic graphite (HOPG) (distance between layers 
$d_{002}=0.335 \mathrm{~nm}$, crystallite size $\left.L=100 \mathrm{~nm}\right)$ and fine crystalline pyrolytic graphite $(\mathrm{FPG})\left(d_{002}=0.340 \mathrm{~nm}, L=30 \mathrm{~nm}\right)$. For both types of graphite there is a significant anisotropy of crystalline structure.

The specimens of graphite intercalation compounds with antimony chloride $\left(\mathrm{SbCl}_{5}\right)$, iodine chloride ( $\left.\mathrm{ICl}\right)$, aluminum chloride $\left(\mathrm{AlCl}_{3}\right)$ and bromine $\left(\mathrm{Br}_{2}\right)$ were obtained with standard two-temperature method. ${ }^{2}$ As it is known, intercalation of graphite results in orderly arrangement of intercalates layers in the graphite matrix and the formation of ordered structure of intercalates layer.

Investigations of structural characteristics of obtained GICs were carried out with x-ray diffraction method. The identity parameters $I_{S}$ were determined from $00 l$-dif-fraction patterns of each GICs specimens and then compounds stages $S$ were calculated with use

$$
I_{S}=d_{S}+(S-1) d_{002}, \quad d_{S}=d_{i}+d_{002},
$$

where $d_{i}$ is the thickness of intercalates layer.

Table 1 presents the synthesis conditions, composition; stages number $s$ and identity period $I_{S}$ for the obtained GICs. In Table 1 also ratios of resistivity at room temperature to resistivity at $T=4.2 \mathrm{~K}\left(\rho_{a 4.2} / \rho_{a 300}\right)$ are shown.

The resistivity along graphite planes $\left(\rho_{a}\right)$ in GICs specimens was measured in temperature interval from $4.2 \mathrm{~K}$ to $293 \mathrm{~K}$ by standard for-probe method. Resistivity measurement error was $0.05 \%$.

Figure 1 presents the temperature dependences of resistivity $\rho_{a}$ for obtained GICs. As it is seen at the figures intercalation leads to a decrease of the resistivity and to change the resistivity temperature coefficient from negative sign in the source graphite on a positive sign in inter-calated graphite. For all specimens of GICs based on FPG in temperature dependence $\rho_{a}(T)$ there is a wide minimum. The exact position and value of minimum vary for different specimens. So for second stage GIC with $\mathrm{SbCl5}$ minimum is observed at $T=38 \mathrm{~K}$, for GIC with $\mathrm{ICl}$ minimum is observed at $T=20 \mathrm{~K}$, while for GIC with $\mathrm{AlCl} 3$ minimum is at $T=87 \mathrm{~K}$ and for GIC with bromine minimum is at $T=150 \mathrm{~K}$. Note, that for GICs based on HOPG in dependence $\rho_{a}(T)$ minimum is not observed at any temperature.

Let us analyze the main mechanisms that determine the temperature dependence of resistivity in the GICs of acceptor type. As you know GICs of acceptor type are twodimensional electronic systems, two-dimensionality of which is due to features of their electronic structure.

TABLE 1. Synthesis conditions, composition, stages number s, identity period $\mathrm{I}_{\mathrm{S}}$ and ratio $\rho_{a 4.2} / \rho_{a 300}$ for GICs specimens.

\begin{tabular}{|c|c|c|c|c|c|}
\hline $\begin{array}{l}\text { Source } \\
\text { graphite }\end{array}$ & Intercalate & $\begin{array}{l}\text { Temperature of } \\
\text { intercalation, } \mathrm{K}\end{array}$ & $I_{S}$ & $S$ & $\left(\rho_{\mathrm{a} 4.2} / \rho_{\mathrm{a} 300}\right)$ \\
\hline HOPG & $\ldots$ & $\ldots$ & 0.335 & $\ldots$ & 1.27 \\
\hline HOPG & $\mathrm{SbCl}_{5}$ & 493 & 1.278 & 2 & 0.56 \\
\hline HOPG & $\mathrm{ICl}$ & 313 & 1.045 & 2 & 0.62 \\
\hline FPG & $\ldots$ & $\ldots$ & 0.340 & $\ldots$ & 1.63 \\
\hline FPG & $\mathrm{SbCl}_{5}$ & 493 & 1.280 & 2 & 0.96 \\
\hline FPG & $\mathrm{ICl}$ & 317 & 1.052 & 1 & 0.92 \\
\hline FPG & $\mathrm{ICl}$ & 317 & 0.712 & 2 & 0.96 \\
\hline FPG & $\mathrm{AlCl}_{3}$ & 483 & 1.306 & 2 & 0.94 \\
\hline FPG & $\mathrm{Br}_{2}$ & 317 & 0.717 & 2 & 1.01 \\
\hline
\end{tabular}

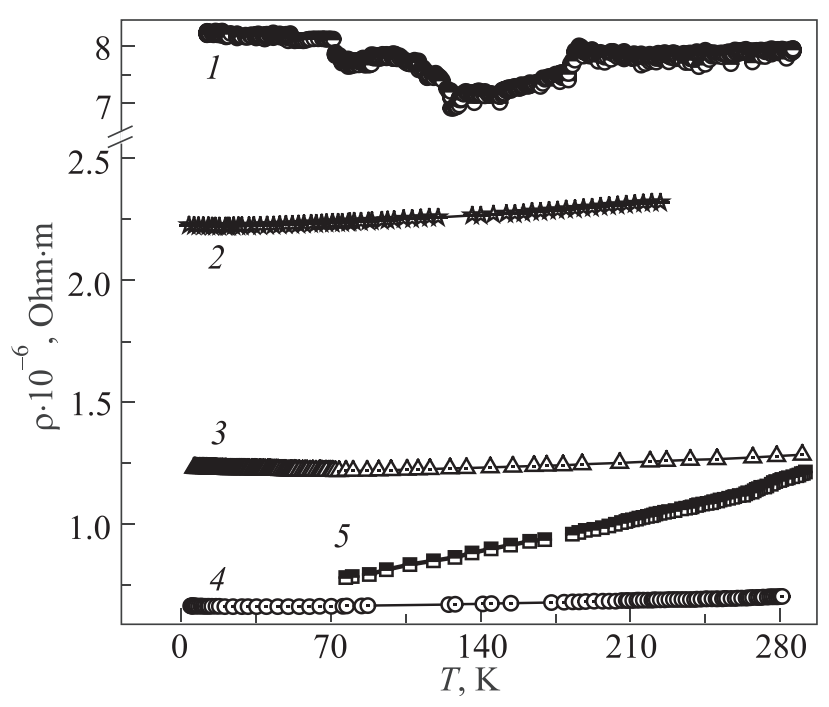

Fig. 1. Experimental dependences $\rho_{a}$ for GICs: $\mathrm{FPG}+\mathrm{Br}_{2}(1)$; $\mathrm{FPG}+\mathrm{ICl}$ (2); $\mathrm{FPG}+\mathrm{AlCl}_{3}(3) ; \mathrm{FPG}+\mathrm{SbCl}_{5}(4)$; and $\mathrm{HOPG}+\mathrm{SbCl}_{5}(5)$.

In the terms of two-dimensional GICs electron structure model of Blinowski and Rigaux ${ }^{14}$ two-dimensional resistivity for two stage compound can be written as

$$
\rho_{2 S a}=\frac{\pi \hbar}{e^{2} k_{F}} \cdot \frac{1}{L_{\mathrm{ef}}},
$$

where $k_{F}$ is the Fermi wave vector, $L_{\mathrm{ef}}$ is the effective mean free path. In approaching of independence of different mechanisms of charge carriers scattering

$$
\frac{1}{L_{\mathrm{ef}}}=\frac{1}{L_{b}}+\frac{1}{L_{T}}
$$

where $L_{b}$ is the free path of the charge carriers scattering on the boundaries of crystallites and $L_{T}$ is the mean free path in the temperature-dependent charge carriers scattering mechanisms. Consider the main carrier scattering mechanisms, that cause the temperature dependence of the electrical resistivity $\rho_{a}(T)$ in GICs. In first, this is electron-electron scattering, which leads to the quadratic temperature dependence of electrical resistivity. ${ }^{15}$ However, according to estimates, resulted in a number of works for the second stage GICs with $\mathrm{SbF}_{5},{ }^{16}$ the mean free path of carriers scattering each other is $7 \times 10^{-5} \mathrm{~m}$ at $100 \mathrm{~K}$. This value is significantly greater than the mean free paths at scattering of charge carriers on phonons and boundaries of crystallites. In second, this is carrier scattering on phonons, which includes scattering on different modes of graphite and intercalate. Thus, in a first approximation the summarized temperature dependence of charge carriers mean free path is determined by the temperature

TABLE 2. Calculated coefficients $A, B, C$ and Fermi energy $E_{F}$ for GICsAs.

\begin{tabular}{lcccc}
\hline \hline GICs & $A, \Omega \mathrm{m} / \mathrm{K}$ & $B, \Omega \mathrm{m}$ & $C$ & $E_{\mathrm{F}}, \mathrm{eV}$ \\
\hline FPG- $\mathrm{SbCl}_{5}$ & $4.37 \times 10^{-12}$ & $6.60 \times 10^{-7}$ & 1.63 & 0.24 \\
$\mathrm{HOPG}-\mathrm{SbCl}_{5}$ & $15.5 \times 10^{-11}$ & $2.27 \times 10^{-7}$ & 1.55 & 0.75 \\
$\mathrm{FPG}-\mathrm{ICl}$ & $1.80 \times 10^{-11}$ & $2.12 \times 10^{-6}$ & 1.64 & 0.11 \\
$\mathrm{FPG}-\mathrm{ICl}$ & $4.55 \times 10^{-11}$ & $1.83 \times 10^{-6}$ & 1.59 & 0.13 \\
FPG- $\mathrm{AlCl}_{3}$ & $8.50 \times 10^{-12}$ & $1.21 \times 10^{-6}$ & 1.60 & 0.19 \\
\hline \hline
\end{tabular}




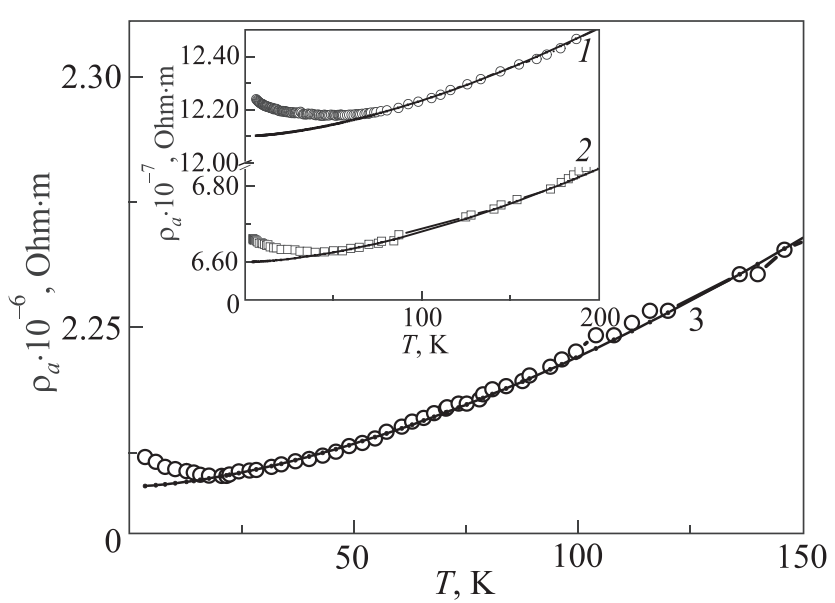

Fig. 2. Experimental $\rho_{a}(T)$ and calculated $\rho_{\text {calc }}$ (corresponding solid lines) dependences for GICs based on FPG with $\mathrm{AlCl}_{3}(1), \mathrm{SbCl}_{5}(2)$ and ICl (3).

dependence of the phonon scattering mean free path, because all other temperature-dependent scattering mean free paths are much larger: $L_{T}=L_{\mathrm{ph}}(T)$. Mean free path of carriers scattering on phonons is inversely proportional to temperature and can be written as

$$
L_{\mathrm{ph}}=L_{0} T^{-c}
$$

where $L_{0}$ and $\mathrm{C}$ are constants.

Thus, the two-dimensional resistivity for second stage GICs in terms of two-dimensional model of the electronic structure can be written as

$$
\rho_{2 S a}=\frac{\pi \hbar}{e^{2} k_{F}} \cdot\left(\frac{1}{L_{b}}+\frac{1}{L_{\mathrm{ph}}(T)}\right)=\frac{3 \pi \hbar b \gamma_{0}}{2 e^{2} E_{F}}\left(\frac{1}{L_{b}}+\frac{1}{L_{\mathrm{ph}}(T)}\right),
$$

where $b$ is the distance between neighboring atoms in a graphite layer, $\gamma_{0}$ is the wave functions overlap integral for neighboring atoms in a graphite layer, $E_{F}$ is the Fermi energy level.

To analyze the temperature dependences of electrical resistivity expression for $\rho_{a}$ taking into account the relations (4) and (5) conveniently presented as

$$
\rho_{a}=A T^{C}+B, \quad A=\frac{\pi \hbar}{e^{2} k_{F}} \cdot \frac{I_{S}}{L_{0}}, \quad B=\frac{\pi \hbar}{e^{2} k_{F}} \cdot \frac{I_{S}}{L_{b}} .
$$

From experimental data on electrical resistivity $\rho_{a}(T)$ for GICs based on structurally different graphites with different intercalates coefficients $A, B, C$ and Fermi energy $E_{F}$ have been calculated. The values of coefficients $A, B, C$ and Fermi energy $E_{F}$ are given in Table 2 .

As it is shown from Table 2 the experimentally determined value of $C=1.6$ for both GIC based on FPG and HOPG. The temperature-independent term $B$ is in 2.5-3.5 times higher for GICs-based on FPG in comparison with GICs based on HOPG while as factor $A$ (factor at $T$ ) is in 10-20 times greater for GICs based in HOPG compared with GICs based on FPG.

Thus, the main mechanisms that determine the temperature dependence of the resistivity $\rho_{\mathrm{a}}$ in the temperature range $60-300 \mathrm{~K}$ for both the GICs based on VOPH and for the
GICs based on FPG are the same. These compounds are characterized by positive resistivity temperature coefficient, which weakly depends on the type of intercalate and significantly decreases with decreasing of crystallite size of the source graphite.

Figure 2 presents the calculated with Eq. (6) temperature dependences of resistivity $\rho_{a}(T)$.

As it is revealed from figure Eq. (6) very well describes the experimental dependence $\rho_{a}(T)$ at the high temperature. While at low temperatures significant deviation from dependence (6) is observed. The minimum on the curves $\rho_{a}(T)$ indicates that for GICs in low-temperature interval conductivity mechanism is different from the classic. Such abnormal temperature dependence of resistivity may be related with effect of weak localization and electron-electron interaction charge carriers that occur for weekly disordered systems $k_{F}=L_{\text {ef }}$. The electrical conductivity with additions due to quantum effects can be written as

$$
\begin{aligned}
\sigma(T) & =\sigma_{\mathrm{cl}}(T)+\delta \sigma_{q}(T), \\
\delta \sigma_{q}(T) & =\delta \sigma_{\text {loc }}(T)+\delta \sigma_{\text {int }}(T),
\end{aligned}
$$

where $\sigma_{\mathrm{cl}}$ is the classic conductivity, $\delta \sigma_{q}$-addition to conductivity due to quantum effects, $\delta \sigma_{q}$ consists the addition due to carriers weak localization effects $\delta \sigma_{\text {loc }}$ and addition due to carriers interaction effects $\delta \sigma_{\text {int }}$. For two-dimensional systems weak localization addition to conductivity $\delta 2 \sigma_{\text {loc }}$ is ${ }^{17-20}$

$$
\delta \sigma_{2 \mathrm{loc}}=\frac{e^{2}}{2 \pi^{2} \hbar} \alpha \ln \left(\frac{\tau_{0}}{\tau_{\varphi}}\right),
$$

and interaction addition $\delta \sigma_{2 \text { int }}$ is

$$
\delta \sigma_{2 \mathrm{int}}=\frac{e^{2}}{2 \pi^{2} \hbar} \gamma \ln \left(\frac{2 \pi k \tau_{0} T}{\hbar}\right)
$$

where $\tau_{0}$ is the charge carriers relaxation time, $\tau_{\varphi}$ is wave function phase relaxation time, $\tau_{\varphi}=A^{*} T^{-P}(p=d / 2, d$ is dimensionality of system $\left.{ }^{21,22}\right) \alpha$ is numerical coefficient that depends on the ratio between $\tau_{\varphi}$ and $\tau_{0} ; \gamma$ is numerical coefficient that reflects the degree of carriers screening. With use Eqs. (7)-(9) addition to two-dimensional conductivity is

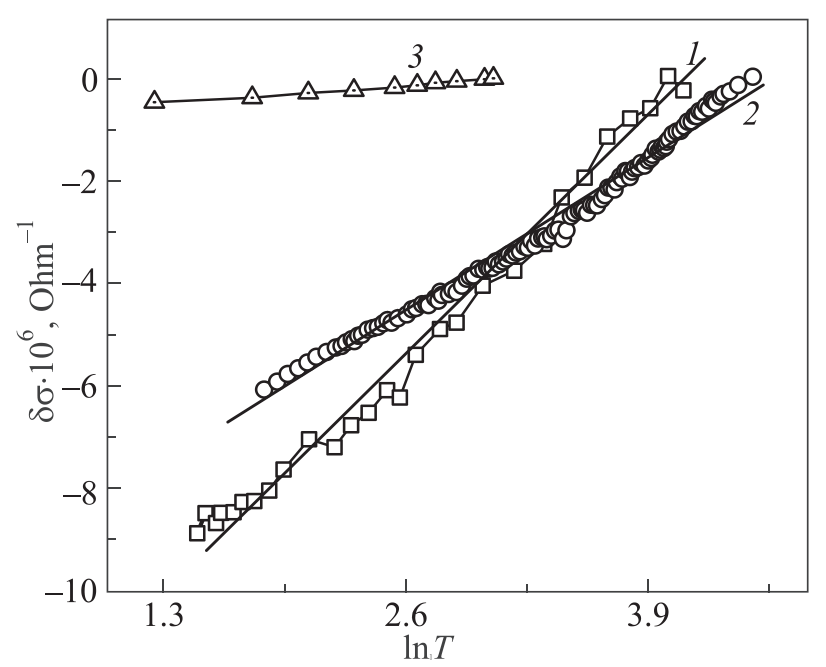

Fig. 3. Dependences $\delta \sigma(T)=\delta \sigma_{2 \exp }(T)-\delta \sigma_{\mathrm{cl}}(T)$ for GICs with ICl (1), $\mathrm{AlCl}_{3}(2)$, and $\mathrm{SbCl}_{5}(3)$. 
TABLE 3. Coefficients $K, E$ and calculated constants $\gamma, \alpha$ and $0 \tau$ for GICs.

\begin{tabular}{|c|c|c|c|c|c|c|c|}
\hline Specimen & $K(\Omega \mathrm{K})^{-1}$ & $E(\Omega)^{-1}$ & $\gamma$ & $\alpha$ & $\tau_{0}, \mathrm{~s}$ & $E_{F}, \mathrm{eV}$ & $\rho_{a \min }, \Omega \mathrm{m}$ \\
\hline $\mathrm{FPG}-\mathrm{SbCl}_{5}$ & $3.48 \times 10^{-6}$ & $-1.445 \times 10^{-5}$ & 0.24 & 0.034 & $2.0 \times 10^{-14}$ & 0.24 & $6.62 \times 10^{-7}$ \\
\hline $\mathrm{FPG}-\mathrm{AlCl}_{3}$ & $2.34 \times 10^{-6}$ & $-1.069 \times 10^{-5}$ & 0.16 & 0.024 & $1.8 \times 10^{-14}$ & 0.19 & $1.21 \times 10^{-6}$ \\
\hline $\mathrm{FPG}-\mathrm{ICl}$ & $2.64 \times 10^{-7}$ & $-8.2 \times 10^{-7}$ & 0.03 & $4.9 \times 10^{-3}$ & $1.5 \times 10^{-14}$ & 0.11 & $2.22 \times 10^{-6}$ \\
\hline
\end{tabular}

$$
\begin{aligned}
\delta \sigma_{2 q}= & \delta \sigma_{2 \operatorname{loc}}+\delta \sigma_{2 \text { int }}=\frac{e^{2}}{2 \pi^{2} \hbar}[(\alpha \mathrm{p}+\gamma) \ln T \\
& \left.+\gamma \ln \left(\frac{2 \pi k_{b} \tau_{0}}{\hbar}\right)+\alpha \ln \left(\frac{\tau_{0}}{A^{*}}\right)\right]
\end{aligned}
$$

or in simplified form

$$
\delta \sigma_{2 q}=K \ln T+E
$$

where

$$
\begin{aligned}
& K=\frac{e^{2}}{2 \pi^{2} \hbar}(\alpha p+\gamma) \\
& E=\frac{e^{2}}{2 \pi^{2} \hbar}\left[\gamma \ln \left(\frac{2 \pi k_{b} \tau_{0}}{\hbar}\right)+\alpha \ln \left(\frac{\tau_{0}}{A^{*}}\right)\right] .
\end{aligned}
$$

Thus, for two-dimensional system additions to conductivity related with manifestation of quantum effects of charge carriers weak localization and interaction are proportional to $\ln T$.

Figure 3 presents the temperature dependence $\delta \sigma(T)$ $=\delta \sigma_{2 \exp }(T)-\delta \sigma_{\mathrm{cl}}(T)$ for GICs specimens, where $\sigma_{2 \exp }=$ $I_{S} /\left(2 \rho_{a \text { exp }}\right)$ according to simple two-dimensional model of GICs electron structure, $\sigma_{\mathrm{cl}}$ is calculated with use Eq. (7).

As it is shown Fig. 3, linear dependence of $\delta \sigma$ from $\ln T$ is observed. Such dependence $\delta \sigma(\ln T)$ indicates the possibility of realization of quantum effects of weak localization and interaction in GICs and the two-dimensional character of conductivity in them. From dependences $\delta \sigma(\ln T)$ the coefficients $K$ and $E$ were determined (Table 3 ) and with use Eq. (11) the constants $\gamma, \alpha$, and $\tau_{0}$ were calculated. For calculation the value of $A^{*}$ is taken as in Ref. 9. Values of constants are in Table 3 . In Table 3 also the values of Fermi energy $E_{F}$ and resistivity in minimum $\rho_{a \text { min }}$ are presented.

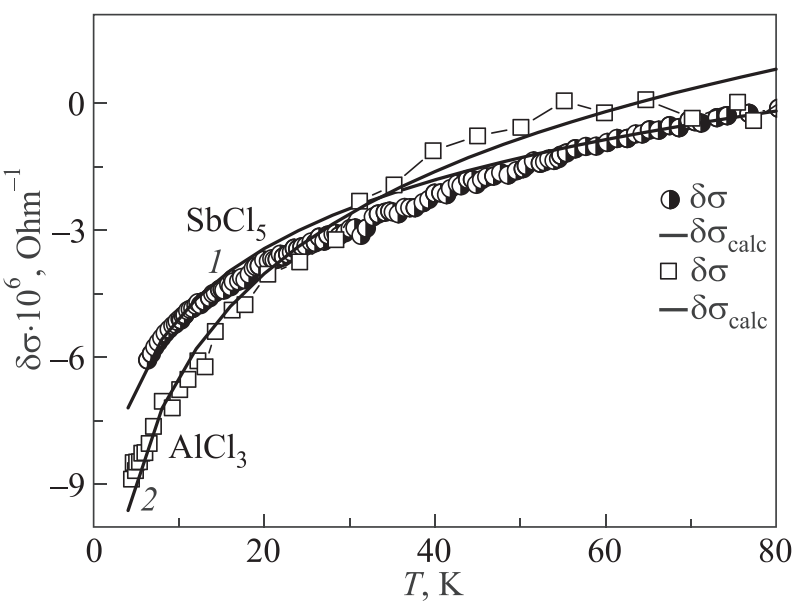

Fig. 4. Calculated temperature dependences of additions to two-dimensional conductivity, $\delta \sigma(T)=\delta \sigma_{2 \exp }(T)-\delta \sigma_{\mathrm{cl}}(T)$ and $\delta \sigma_{\text {calc }}(T): \mathrm{FPG}-\mathrm{SbCl}_{5}(1)$ and $\mathrm{FPG}-\mathrm{AlCl}_{3}(2)$.
As can be seen from the table, for all specimens of GICs constant $\gamma$, which is related with shielding of charge carriers at the interaction, exceeds the constant $\alpha$ that is responsible for weak localization, about 6-7 times.

Figure 4 presents the calculated according to (9) addition to conductivity $\delta \sigma_{2 q}$ with use calculated parameters $\gamma, \alpha$, and $\tau_{0}$.

As is shown at Fig. 4 calculated addition $\delta \sigma_{2 q}$ well coincides with the experimentally determined $\delta \sigma(T)=\delta \sigma_{2 \exp }(T)$ $-\delta \sigma_{\mathrm{cl}}(T)$.

\section{Conclusions}

Thus, the revealed low-temperature anomalies in the resistivity of acceptor type GICs based on FPG can be explained as for source for intercalation FPG in the terms of theory of charge carriers weak localization and interaction effects. For GICs the additions to conductivity due to weak localization and interaction are two-dimensional in contrast to the three-dimensional additions to conductivity for source for intercalation GPG. The comparative analysis of the calculated values of shielding constants $\gamma$ and weak localization constants $\alpha$ for GICs with different acceptor intercalates allows us to conclude that for low stage GICs based on FPG low-temperature peculiarities in the conductivity caused mainly by effect of the charge carriers interaction. For GICs based on HOPG the low-temperature anomalies in the conductivity were not revealed.

${ }^{a)}$ Email: matzui@univ.kiev.ua

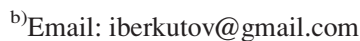

${ }^{1}$ B. L. Al'tshuler, D. Khmel'nitzkii, A. I. Larkin, and P. A. Lee, Phys. Rev. B 22, 5142 (1980).

${ }^{2}$ B. L. Al'tshuler, A. G. Aronov, A. I. Larkin, and D. E. Khmel'nitskii, Zh. Eksp. Teor. Fiz. 81, 768 (1981) [Sov. Phys. JETP 54, 411 (1981)].

${ }^{3}$ B. L. AI'tshuler and A. G. Aronov, Zh. Eksp. Teor. Fiz. 77, 2028 (1979) [Sov. Phys. JETP 50, 968 (1979)].

${ }^{4}$ Y. Y. Charkov, L. L. Kolesnichenko, and L. Yu. Matzui, Solid State Phys. 25, 594 (1983).

${ }^{5}$ L. Yu. Matzui and A. M. Katsiuba, Bull. Kiev Univ., Ser.: Phys. Math. 1, 342 (1999).

${ }^{6}$ I. V. Ovsienko, T. A. Len, L. Yu. Matzui, Yu. I. Prylutskyy, I. B. Berkutov, V. V. Andrievskii, Yu. F. Komnik, I. G. Mirzoiev, G. E. Grechnev, Yu. A. Kolesnichenko, R. Hayn, and P. Scharff, Phys. Status Solidi 252, 1402 (2015).

${ }^{7}$ I. V. Ovsienko, L. Yu. Matzui, I. V. Yatsenko, S. V. Khrapatiy, Yu. I. Prylutskyy, U. Ritter, P. Scharff, and F. Normand, Materialwiss. Werkstofftech. 44, 161 (2013).

${ }^{8}$ L. Yu. Matzui, I. V. Ovsienko, T. A. Len, Yu. I. Prylutskyy, and P. Scharff, Fullerenes Nanotubes Carbon Nanostruct. 13, 259 (2005).

${ }^{9}$ T. A. Len, L. Yu. Matzui, I. V. Ovsienko, Yu. I. Prylutskyy, V. V. Andrievskii, I. B. Berkutov, G. E. Grechnev, and Yu. A. Kolesnichenko, Fiz. Nizk. Temp. 37, 1027 (2011) [Low Temp. Phys. 37, 819 (2011)].

${ }^{10}$ L. Piraux, V. Bayot, J. P. Michenaud, and J. P. Issi, Solid State Commun. 59, 711 (1986).

${ }^{11}$ L. Piraux and V. Bayot, Phys. Rev. B 36, 9045 (1987).

${ }^{12}$ L. Piraux, V. Bayot, and M. S. Dresselhaus, Phys. Rev. B 45, 14315 (1992). 
${ }^{13}$ L. Piraux, V. Bayot, J. P. Michenaud, and J. P. Issi, Phys. Scr. 37, 942 (1988). ${ }^{14}$ J. Blinowski and C. Rigaux, J. Phys. 41, 667 (1980).

${ }^{15}$ K. Matsubara, K. Sugihara, I.-S. Suzuki, and M. Suzuki, J. Phys. Condens. Matter 11, 3149 (1999).

${ }^{16}$ L. Piraux, K. Amine, V. Bayot, and J. P. Issi, Mater. Sci. Forum 91-93, 481 (1992).

${ }^{17}$ B. L. Al'tshuler and A. G. Aronov, Solid State Commun. 46, 429 (1983).

${ }^{18}$ K. Shimakawa, K. Hayashi, and T. Kameyama, Philos. Mag. Lett. 64, 375 (1991).
${ }^{19}$ A. K. Savchenko, V. N. Lutskii, and A. S. Rypik, Lett. JETP 34, 367 (1981).

${ }^{20}$ M. Kaveh, Can. J. Phys. 60, 746 (1982)

${ }^{21}$ P. W. Anderson, Phys. Rev. 109, 1492 (1958).

${ }^{22}$ D. J. Thouless, Physica B 109, 1523 (1982).

This article was published in English in the original Russian journal. Reproduced here with stylistic changes by AIP Publishing. 